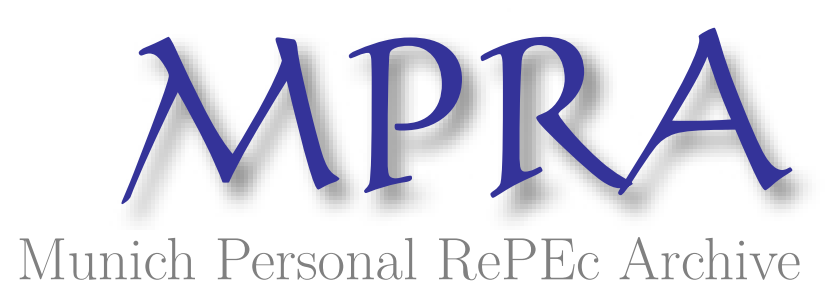

Market Procyclicality and Systemic Risk

Tasca, Paolo and Battiston, Stefano

Swiss Federal Institute of Technology

March 2013

Online at https://mpra.ub.uni-muenchen.de/45156/

MPRA Paper No. 45156, posted 17 Mar 2013 03:31 UTC 


\section{ETH}

Eidgenössische Technische Hochschule Zürich Swiss Federal Institute of Technology Zurich

http://www.sg.ethz.ch
P. Tasca, S. Battiston:

Market Procyclicality and Systemic Risk

Submitted March 16, 2013

\title{
Market Procyclicality and Systemic Risk
}

\author{
Paolo Tasca, Stefano Battiston
}

Chair of Systems Design, ETH Zurich, Weinbergstrasse 58, 8092 Zurich, Switzerland

\begin{abstract}
We model the systemic risk associated with the so-called balance-sheet amplification mechanism in a system of banks with interlocked balance sheets and with positions in realeconomy-related assets. Our modeling framework integrates a stochastic price dynamics with an active balance-sheet management aimed to maintain the Value-at-Risk at a target level. We find that a strong compliance with capital requirements, usually alleged to be procyclical, does not increase systemic risk unless the asset market is illiquid. Conversely, when the asset market is illiquid, even a weak compliance with capital requirements increases significantly systemic risk. Our findings have implications in terms of possible macro-prudential policies to mitigate systemic risk.
\end{abstract}

Keywords: Systemic risk, Procyclicality, Leverage, Market liquidity, Network models JEL classification: G20, G28

\section{Introduction}

In a mark-to-market financial system, asset price movements have an instantaneous impact on the net worth of the market participants who hold those assets. This effect is amplified when financial institutions use borrowing to leverage their exposure to risky assets. Moreover, there are also indirect spillover effects on market participants who hold claims on those holding the assets. Therefore, aggregate asset-price shocks can propagate through the financial system via both direct and indirect linkages. A recent body of research has investigated the relation between price changes and subsequent balance sheet adjustments resulting from the fact that institutions actively manage their Value-at-Risk (Adrian and Shin, 2010, 2011b; Shin, 2008). This practice leads to spirals of asset devaluation (or overvaluation). Accordingly, a debate has developed regarding the appropriate policy instruments to use to mitigate the procyclical effects arising

\footnotetext{
${ }^{0}$ We are grateful to Andrea Collevecchio, Martino Grasselli, Iman Lelyveld, Loriana Pelizzon, Paolo Pellizzari, Frank Schweitzer, Jean-Charles Rochet, Didier Sornette, Claudio J. Tessone. The authors acknowledge financial support from the ETH Competence Center "Coping with Crises in Complex Socio-Economic Systems" CHIRP 1 (grant no. CH1-01-08-2), the European Commission FET Open Project "FOC" (grant no. 255987), and the Swiss National Science Foundation project "OTC Derivatives and Systemic Risk in Financial Networks" (grant no. CR12I1-127000/1). Correspondence to Paolo Tasca, ptasca@ethz.ch.
} 


\section{ETH}

Eidgenössische Technische Hochschule Zürich Swiss Federal Institute of Technology Zurich

http: //www.sg.ethz.ch
P. Tasca, S. Battiston:

Market Procyclicality and Systemic Risk

Submitted March 16, 2013

from the interplay between leverage and mark-to-market asset valuation (see e.g., BIS, 2010; EC, 2011).

Our goal is to asses how systemic risk depends on the interaction between (1) the level of bank compliance with capital requirements and (2) asset market liquidity in the presence of an unexpected common price shock. ${ }^{1}$ Unlike previous works focusing on price volatility, we characterize risk in terms of time-to-default in a system context by combining a balance sheet approach with a dynamic stochastic framework.

First, we model a financial system that is composed of leveraged financial institutions (hereafter, "banks") that manage diversified but possibly overlapping portfolios of real-economy-related assets (hereafter, "external assets"). Banks hold claims on each other (hereafter, "interbank claims"), the value of which depend on the obligor's leverage. Finally, banks borrow funds from market players outside the banking network (hereafter, "external funds"). Second, we model dynamic balance-sheet management. As noted by Adrian and Shin (2010), the practice of adjusting the Value-at-Risk (VaR) to a given target level is equivalent to maintaining leverage close to a constant value of financial reporting leverage (hereafter, "target leverage"). The paper formalizes an accounting rule in support of Adrian and Shin (2010) findings, whereby banks sell or buy external assets in response to price movements. An increase (decrease) in prices induces an expansion (contraction) of the balance sheets, which generates further price movements in the same direction. Consequently, prices exhibit a stochastic dynamics whose return is influenced by bank trades. In other words, the combination of (1) balance-sheet management and (2) the price response generates a positive feedback loop between leverage and prices (i.e., leverage-price cycle) that may amplify the effects of common shocks into a spiral of asset price devaluation or over-valuation.

We thus introduce a table of market procyclicality in the exogenous parameters $\varepsilon$ and $\gamma$. The parameter $\varepsilon$ represents the level of bank compliance with capital requirements, i.e., promptness in adjusting leverage deviations from the target level. Values of $\varepsilon$ that are close to zero denote banks' sluggish adjustments, whereas values of $\varepsilon$ that are close to one mean that banks react very promptly. In other words, $\varepsilon$ can be understood as a scaling factor of the trading size engendered by the accounting rule. The parameter $\gamma$ captures the market impact (i.e., the average price response to bank trades). $\gamma$ is also related to the market liquidity risk. We define a liquid market as a market where participants can execute large transactions at short notice with minimal impact on the price. An asset will be liquid if it is traded in a liquid market. Therefore, low (high) values of $\gamma$ imply a liquid (illiquid) market. Thus, the market is said to be weakly (strongly) procyclical when both $\varepsilon$ and $\gamma$ are small (large).

\footnotetext{
"'Systemic risk" is meant here as the risk of a systemic default and in the following it will be used interchangeably with the term "probability of systemic default".
} 


\section{ETH}

Eidgenössische Technische Hochschule Zürich Swiss Federal Institute of Technology Zurich

http://www.sg.ethz.ch
P. Tasca, S. Battiston:

Market Procyclicality and Systemic Risk

Submitted March 16, 2013

Although the model can accommodate various market microstructures and heterogeneous players, in the analysis we focus on a scenario that includes a homogeneous and tightly-knit financial system. In this context, we test the resilience of the system by introducing a common market-wide price shock (hereafter, "price shock"). The price shock is generic because we do not distinguish between fundamental and non-fundamental shocks. In addition, the asset market is incomplete in the sense that banks cannot insure themselves against the price shock (i.e., there are no Arrow securities that are contingent on the shock).

Our findings are as follows. A negative shock to asset prices depletes capital and increases leverage. When $\varepsilon$ is not too small and $\gamma$ is sufficiently high, (i.e., when the price response to bank trades is more rapid than the adjustment in leverage), banks continue pursuing their target leverage without reaching it. In so doing, banks generally amplify the effect of the initial price shock. Therefore, the probability of systemic default is higher. Conversely, when $\varepsilon$ is high and $\gamma$ is very close to zero (i.e., when the market is so liquid that the price response to bank trades is considerably weaker than the adjustments in leverage), banks manage to promptly track their target leverage without triggering an amplification of the initial price shock. In this case, the probability of systemic default remains low. In addition, the mix of funding sources is not irrelevant: the probability of systemic default increases with the average bank exposures to external funds relative to interbank funds.

Overall, this paper sheds light on the mechanisms that govern the emergence of the tension between (1) the individual incentive to target a given VaR (relative to its economic capital) and (2) systemic risk. In terms of their policy implications, our results are significant because they suggest that in the presence of price shocks, counter-cyclical policies should take into account the interplay between banks' procyclical behavior and the time-variation of asset market liquidity.

This paper is structured as follows. We begin with a review of the related literature. Then, Section 2 introduces the model. In Section 3, we simplify the modeling framework using meanfield approximation and conduct a numerical analysis of the probability of systemic default. Section 4 presents the results. Section 5 concludes and considers the policy implications of the results.

\subsection{Related Literature}

This paper is related to the following discussions in the literature. Several authors have investigated financial contagion in the interbank market (see e.g., Allen and Gale, 2001; Battiston et al., 2012a,b; Elsinger et al., 2006; Freixas et al., 2000; Furfine, 2003; Tasca and Battiston, 2011). Other studies have investigated contagion effects mediated by common asset holding (see e.g., Allen et al., 2012; Kiyotaki and Moore, 2002). As our paper adopts a balance-sheet approach, the results are pertinent to the literature on the amplification of financial shocks via balance sheet 


\section{ETH}

Eidgenössische Technische Hochschule Zürich Swiss Federal Institute of Technology Zurich

http://www.sg.ethz.ch
P. Tasca, S. Battiston:

Market Procyclicality and Systemic Risk

Submitted March 16, 2013

transmission mechanisms (Brunnermeier, 2008; Kashyap and Stein, 2000) - a literature review on this topic is carried out in Krishnamurthy (2010). Moreover, the paper offers a first attempt to link the balance-sheet approach to a dynamic stochastic setting in a network context. Using this framework, we model the procyclicality of leverage, the empirical evidence of which has been documented mainly in Adrian and Shin (2008a, 2010) and Greenlaw et al. (2008). In particular, we study the macro-level consequences of procyclical leverage and analyze the mutual influence between the contraction and expansion of banks' balance sheets and asset prices. In a different setting, Adrian and Shin (2011b) offer a two-stage principal-agent model for use in studying the relationship between balance sheet leverage and the riskiness of bank assets. Instead, our network approach is more related to the recent literature on the network-based "leverage accelerator" mechanism (see e.g., Bargigli et al., 2012). Moreover, as liquidity risk is embedded in our model, the paper complements a growing literature on funding and market liquidity problems that in the recent U.S. crisis (2007-2009) started the systemic liquidity spirals (see e.g., Gârleanu and Pedersen, 2007). Our model partially resorts to the assumptions of "equity rationing"(see e.g., Stiglitz and Weiss, 1981) and "debt overhang", the idea that, under certain conditions, it is difficult or inconvenient for banks to raise new equity; evidence and possible causes are discussed in Adrian and Shin (2011a). Moreover, the idea that capital scarcity may lead to a "credit crunch" has been investigated by Bernanke et al. (1991); Calomiris and Wilson (1998), among others. Finally, the problem of the procyclical effects of capital adequacy regulation is a classic conflict between the micro and macro perspectives on financial stability (see e.g., Acharya, 2009). This issue has been widely discussed in the context of the Basel accords (see e.g., Blum and Hellwig, 1995; Estrella, 2004).

\section{Model}

\subsection{The Interbank Network}

Let time be indexed as $t \in[0, T]$, and consider an interbank market composed of a set $\Omega_{n}$ of $n$ risk-averse banks bound by market risk-based capital requirements. To keep the notation simple, whenever it is unambiguous given the context that a variable is time-dependent, we will omit the index $t$. The balance-sheet identity of bank $i \in \Omega_{n}$ is given by

$$
a_{i}=h_{i}+b_{i}+e_{i} \text {. }
$$

where $a_{i}$ is the market value of bank $i$ 's assets, $h_{i}$ is the book value of bank $i$ 's obligations to other banks in the network (e.g., zero-coupon bonds), ${ }^{2} b_{i}$ is the book value of bank $i$ 's external

\footnotetext{
${ }^{2}$ More specifically, $h_{i}$ is the value of the payment at maturity that each obligor $i$ has promised to its lenders. The equation implicitly assumes that, for all of the banks in the system, there is only one type of debt with the same maturity date and seniority.
} 


\section{ETH}

Eidgenössische Technische Hochschule Zürich Swiss Federal Institute of Technology Zurich

http://www.sg.ethz.ch
P. Tasca, S. Battiston:

Market Procyclicality and Systemic Risk

Submitted March 16, 2013

funds with actors outside the banking system, and $e_{i}$ is the equity value. It is assumed that each bank invests in two asset classes composed of: (i) $n-1$ obligations, each issued by one of the other banks in the system and (ii) $m$ homogeneous investment opportunities that belong to the set $\Omega_{m}$ of external assets related to the real side of the economy. Hereafter, for the sake of simplicity, we omit the lower and upper bounds of the summations. It remains understood that, in the summation for external assets, the index ranges from 1 to $m$, and that, in the summation for banks, the index ranges from 1 to $n$. The asset side in Eq. (1) reads

$$
a_{i}:=\sum_{l} Q_{i l} s_{l}+\sum_{j} W_{i j} \hbar_{j} .
$$

In the equation above, $Q_{i l}(\geq 0)$ is the quantity of the external asset $l$ held by $i ; s_{l}$ is the price of the external assets $l ; W_{i j}(\geq 0)$ (with $W_{i i}=0$ ) is the quantity of debt issued by $j$ and held by $i$; and $\hbar_{j}$ is the present market value of bank $j$ 's debts, defined as the discounted value of future payoffs:

$$
\hbar_{j}:=h_{j}\left[1+r_{j}\right]^{-\hat{t}},
$$

where $r_{j}$ is the rate of return on $\hat{t}$-years maturity obligations, which are assumed to be continuously rolled over at every time $t$. Correspondingly, bank $i$ 's balance sheet can be represented as follows.

Bank- $i$ balance-sheet
\begin{tabular}{|c|c|}
\hline Assets & Liabilities \\
\hline$\sum_{j} Q_{i l} s_{l}$ & $h_{i}$ \\
$\sum_{j} W_{i j} \hbar_{j}$ & $b_{i}$ \\
& $e_{i}$ \\
\hline
\end{tabular}

Fig. (1) illustrates one possible architecture of the financial system described in our model. In general, there can be overlaps among banks' portfolios of external assets, external funds and interbank claims. For instance, banks 1 and 2 have one external asset in common, and both hold claims against bank 4 . Bank 4 shares the same external fund with bank 3. 


\section{ETH}

Eidgenössische Technische Hochschule Zürich Swiss Federal Institute of Technology Zurich

http://www.sg.ethz.ch
P. Tasca, S. Battiston:

Market Procyclicality and Systemic Risk

Submitted March 16, 2013
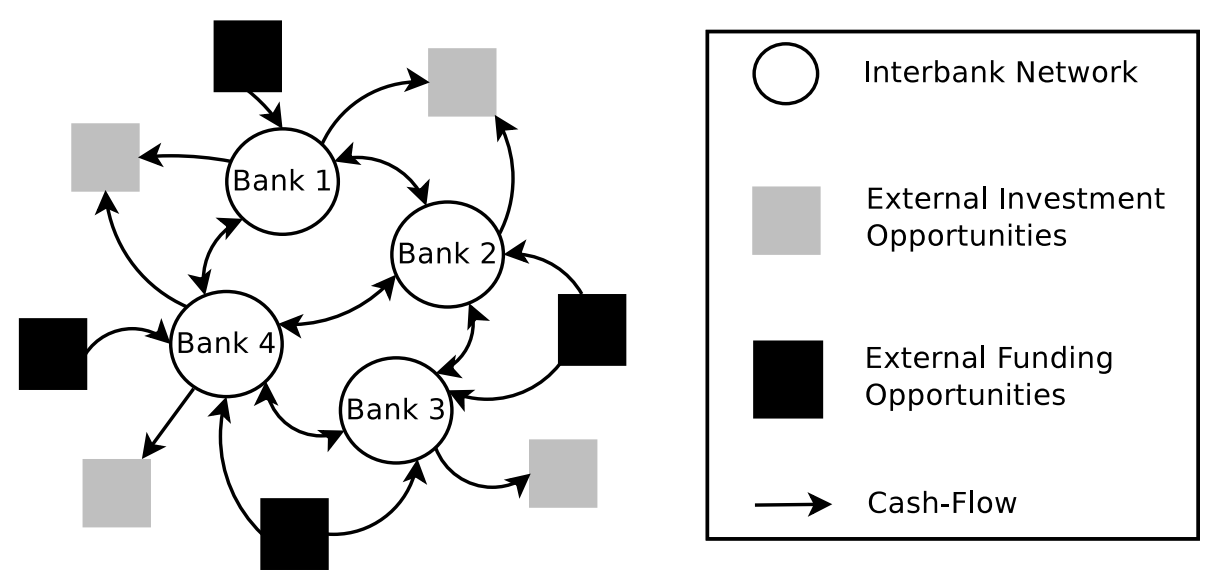

Figure 1: Illustration example of a stylized architecture of the financial system.

\subsection{Leverage}

The bank $i$ 's leverage is defined as its debt-asset ratio. ${ }^{3}$ From Eq. (1) and Eq. (2) we have,

$$
\begin{aligned}
\phi_{i} & :=\left(h_{i}+b_{i}\right) / a_{i} \\
& =\left(h_{i}+b_{i}\right) /\left(\sum_{l} Q_{i l} s_{l}+\sum_{j} W_{i j} \hbar_{j}\right) \\
& =\left(h_{i}+b_{i}\right) /\left(\sum_{l} Q_{i l} s_{l}+\sum_{j} W_{i j} h_{j}\left[1+r_{j}\right]^{-\hat{t}}\right) \in(0,1] .
\end{aligned}
$$

We assume that the rate of return on claims on other banks depends linearly upon the obligor's credit worthiness as measured by its leverage. In particular, as in Tasca and Battiston (2011), we assume that for each obligor $j$, the following relationship holds:

$$
r_{j}:=r_{f}+\beta \phi_{j},
$$

where $r_{f}$ is the risk-free rate, and where $\beta \phi_{j}$ denotes the credit spread associated with the $j$ issuer. The parameter $\beta \in(0,1)$ is the factor loading on $j$ 's leverage and can be understood as the responsiveness of the rate of return to the bank's financial condition. After substituting Eq. (4) into Eq. (3c), we obtain a system of coupled equations in which bank $i$ 's leverage is a positive function of the leverage of all of the obligors of $i$ :

$$
\phi_{i}=\left(h_{i}+b_{i}\right) /\left(\sum_{l} Q_{i l} s_{l}+\sum_{j} W_{i j} h_{j}\left[1+r_{f}+\beta \phi_{j}\right]^{-\hat{t}}\right) .
$$

\footnotetext{
${ }^{3}$ Notice that the leverage is always bigger than zero since banks are by definition debt-financed.
} 


\section{ETH}

Eidgenössische Technische Hochschule Zürich Swiss Federal Institute of Technology Zurich

http://www.sg.ethz.ch
P. Tasca, S. Battiston:

Market Procyclicality and Systemic Risk

Submitted March 16, 2013

\subsection{Target Leverage. An Accounting Rule}

The VaR is widely considered by financial institutions as part of their risk management procedure. As in Shin (2008), we assume that each bank $i \in \Omega_{n}$ adjusts its balance sheet to maintain "economic capital" (equity $e_{i}$ ) equal to its VaR. In other words, the VaR is the equity capital that a bank must hold to remain solvent with probability $c$. As explained below, this practice is equivalent to targeting a reference leverage $\phi_{i}^{*}$. Let us use $V_{i}$ to denote the VaR per dollar of assets held by $i$. If bank $i$ maintains equity capital $e_{i}$ to meet total VaR, then we have $e_{i}=V_{i} \times a_{i} \Rightarrow a_{i}-h_{i}+b_{i}=V_{i} \times a_{i} \Rightarrow h_{i}+b_{i}=a_{i} \times\left(1-V_{i}\right)$. This implies that $i$ has a target leverage $\phi_{i}^{*}=\left(h_{i}+b_{i}\right) / a_{i}=\left(1-V_{i}\right)$.

The accounting rule that allows bank $i$ to keep its leverage close to $\phi_{i}^{*}$ over time finds its economic ground in Adrian and Shin (2008a, 2010) and operates as follows. In a falling market (i.e., under slumping external asset prices), the leverage increases (i.e., $\phi_{i}>\phi_{i}^{*}$ ). In this scenario, the bank shrinks its balance sheet by selling (a portion of) its external assets and paying back (a portion of) its external debts with the proceeds. In a rising market (i.e., under booming external asset prices), the leverage decreases (i.e., $\phi_{i}<\phi_{i}^{*}$ ). In such a scenario, the bank expands its balance sheet by taking on additional external funds to invest the "fresh" cash in external activities. Therefore, the dynamics of banks' balance sheets may reinforce cyclical upturns and downturns. To derive a formal accounting rule, we make the following assumptions:

1. Bank $i$ can neither issue new equity or replace equity with debts. ${ }^{4}$

2. The notional value of bank $i$ 's interbank debts is constant over time, i.e., $h_{i}(t)=h_{i}$ for all $t \geq 0 .{ }^{5}$

Note that the second assumption does not prevent bank $i$ from changing counterparties or adjusting their relative shares in $i$ 's interbank debts. Moreover, it does not prevent the market value of the interbank claims from changing over time based on the time-varying credit worthiness of the debt issuer (see Eq. 4). The two assumptions above, together with the balance sheet identity in Eq. (1), imply that any variation in the external assets held by bank $i$ will correspond to an equal amount of variation in the external funds on the liability side.

Formally, under the accounting constraints $Q_{i}=\sum_{l} Q_{i l} ; d Q_{i} \geq-Q_{i} ; d b_{i} \geq-b_{i}$, we obtain the following accounting rule (whose derivation is provided in Appendix A).

$$
\frac{d b_{i}}{b_{i}}=\left(\frac{\varepsilon_{i}}{\kappa_{i} \phi_{i}}\right)\left(\frac{\phi_{i}^{*}-\phi_{i}}{1-\phi_{i}^{*}}\right)
$$

\footnotetext{
${ }^{4}$ Some candidate explanations in support of the fact that equity remains "sticky" are explained in Adrian and Shin (2011a).

${ }^{5}$ Because our aim is to model the leverage-price cycle, this hypothesis allows us to better capture the spillover effect on the real economy that results from the re-adjustments of banks' claims against real-economy-related activities.
} 


\section{ETH}

Eidgenössische Technische Hochschule Zürich Swiss Federal Institute of Technology Zurich

http://www.sg.ethz.ch
P. Tasca, S. Battiston:

Market Procyclicality and Systemic Risk

Submitted March 16, 2013

$$
\frac{d Q_{i l}}{Q_{i l}}=\left(\frac{\varepsilon_{i}}{\alpha_{i l}}\right)\left(\frac{\phi_{i}^{*}-\phi_{i}}{1-\phi_{i}^{*}}\right) .
$$

The parameter $\varepsilon_{i} \in(0,1]$ measures the promptness of $i$ in pursuing the target level of leverage $\phi_{i}^{*}$. It can also be seen as a scaling factor of $i$ 's trading size. The parameter $\kappa_{i}:=b_{i} /\left(b_{i}+h_{i}\right) \in(0,1]$ is the ratio of external funds to total debts, and $\alpha_{i l}:=Q_{i l} s_{l} /\left(\sum_{l} Q_{i l} s_{l}+\sum_{j} W_{i j} \hbar_{j}\right) \in(0,1]$ is the ratio of the external asset $l$ to total assets.

\subsection{Leverage-Price Cycle and Market Liquidity}

In this section, we formalize the leverage-price cycle and link it to the notion of market liquidity. Inspired by the empirical research on the leverage-asset price cycle (Adrian and Shin, 2008b, 2010), we now model how the accounting rule in Eq. (7) impacts the price of (non-payingdividend) external assets whose dynamics are driven by a standard $\mathrm{GBM}^{6}$

$$
\frac{d s_{l}}{s_{l}}=\mu_{l} d t+\sigma_{l} d B_{l}, \quad \forall l \in \Omega_{M} .
$$

The (bid-ask) trading sizes serve as measures of the strength of the (demand-supply) pressures on external assets at a given time. A large bid size indicates a strong demand for the external asset. A large ask size shows that there is a large supply of the external asset. Banks act as "active"traders because they demand immediacy and push prices in the direction of their trading. Instead, players outside the banking system act as "passive" traders who act as liquidity providers. These players are "contrarian" who take the opposite side of the banks' transactions: they sell when the price is high and buy when the price is low. For a given external asset $l \in \Omega_{m}$ with trading volume $Q_{l}=\sum_{i} Q_{i l}$, if banks place a bid on the order book, then the demand for the asset is larger than the supply (i.e., $d Q_{l}>0$ ); therefore, the price $s_{l}$ is likely to go up. If banks place an ask on the order book, then the supply of the asset is larger than the demand (i.e., $d Q_{l}<0$ ); therefore, the price $s_{l}$ is likely to drop. In order to isolate the non-linear impact of the dynamic balance-sheet management on the asset price dynamics in Eq. (8), we consider a linear relationships between asset returns and trading volume:

$$
\mathbb{E}\left(\frac{d s_{l}}{s_{l}}\right)=\gamma_{l}\left(\frac{d Q_{l}}{Q_{l}}\right)
$$

The parameter $\gamma_{l} \geq 0$ captures the market impact, i.e., the average price response to trade size. This concept is closely related to the demand elasticity of price and is typically measured as

\footnotetext{
${ }^{6}$ Where $B_{l} \sim \mathrm{N}(0, d t)$ is a standard Brownian motion defined on a complete filtered probability space $\left(\Omega ; \mathcal{F} ;\left\{\mathcal{F}_{t}\right\} ; \mathbb{P}\right)$ where $\mathcal{F}_{t}=\sigma\{B(s): s \leq t\}, \mu_{l}$ is the instantaneous risk-adjusted expected growth rate, $\sigma_{l}>0$ is the volatility of the growth rate and $d\left(B_{l}, B_{k}\right)=\rho_{l k}$.
} 


\section{ETH}

Eidgenössische Technische Hochschule Zürich Swiss Federal Institute of Technology Zurich

http://www.sg.ethz.ch
P. Tasca, S. Battiston:

Market Procyclicality and Systemic Risk

Submitted March 16, 2013

the price return following a transaction of a given volume. In other words, it is the effect that market participants have on price when they buy or sell an external asset. It is the extent to which buying or selling moves the price against the buyer or seller, i.e., upward during buying and downward during selling. Market liquidity (as we will refer to it here) measures the size of the price response to trades and is inversely proportional to the scale of the market impact: $1 / \gamma_{l}{ }^{7}$ If trading a given quantity only produces a small price change, the market is said to be liquid. If the trade produces a substantial price change, the market is said to be illiquid. Combining Eq. (8) and Eq. (9), we can rewrite the price dynamics as follows:

$$
\frac{d s_{l}}{s_{l}}=\gamma_{l}\left(\frac{d Q_{l}}{Q_{l}}\right) d t+\sigma_{l} d B_{l}, \quad \forall l \in \Omega_{M} .
$$

Eq. (10), together with Eq. (6)-(7), represents the leverage-price cycle. We can describe this positive feedback loop between leverage and prices as follows. At the beginning, the leverage $\phi_{i}$ is set equal to the target $\phi_{i}^{*}$ at a given "reference" price $s_{l}^{*}$ (see Eq. 5). Therefore, any deviation of $s_{l}$ from $s_{l}^{*}$ leads to a deviation of $\phi_{i}$ from $\phi_{i}^{*}$, which indirectly results from the deviation of $e_{i}$ from $e_{i}^{*}$. This event triggers the previously described accounting rule. When $e_{i}>e_{i}^{*}\left(e_{i}<e_{i}^{*}\right)$, the bank has a "surplus" ("deficit") of capital to allocate to external assets. More specifically, when prices rise (i.e., $s_{l}>s_{l}^{*}$ ), the upward adjustment in leverage entails the purchase of external assets. The greater (aggregate) demand for external assets tends to put upward pressure on prices, and the reference price increases. In a downtrend, this mechanism is reversed. In a downtrend market (i.e., $s_{l}<s_{l}^{*}$ ), the downward adjustment of leverage to the target level entails (forced) sales of external assets. ${ }^{8}$ The lower (aggregate) demand for external assets puts downward pressure on prices, and the reference price decreases.

\subsection{The Financial System in a Nutshell}

In summary, the dynamics of the financial market can be described by the following $3 \times n+m$ system of coupled equations with indeces range as follows $l=1, \ldots, m ; i=1, \ldots, n$, and parameter values reported in Table 1.

$$
\begin{cases}\frac{d s_{l}}{s_{l}}= & \gamma_{l}\left(\frac{d Q_{l}}{Q_{l}}\right) d t+\sigma d B_{l} \\ \frac{d Q_{i l}}{Q_{i l}}= & \left(\frac{\varepsilon_{i}}{\alpha_{i l}}\right)\left(\frac{\phi_{i}^{*}-\phi_{i}}{1-\phi_{i}^{*}}\right) d t \\ \frac{d b_{i}}{b_{i}}= & \left(\frac{\varepsilon_{i}}{\kappa_{i} \phi_{i}}\right)\left(\frac{\phi_{i}^{*}-\phi_{i}}{1-\phi_{i}^{*}}\right) d t \\ \phi_{i}= & \left(h_{i}+b_{i}\right) /\left(\sum_{l} Q_{i l} s_{l}+\sum_{j} W_{i j} \frac{h_{j}}{\left(1+r_{f}+\beta \phi_{j}\right)^{t}}\right) .\end{cases}
$$

\footnotetext{
${ }^{7}$ For the sake of simplicity, in our model, $\gamma_{l}$ does not change with trading volume.

8 This "flight to quality" process leads to a price decrease, which in turn forces further selling and further price decreases.
} 


\section{ETH}

Eidgenössische Technische Hochschule Zürich Swiss Federal Institute of Technology Zurich

http://www.sg.ethz.ch
P. Tasca, S. Battiston:

Market Procyclicality and Systemic Risk

Submitted March 16, 2013

In this scheme, procyclicality is caused by a chain reaction triggered by an exogenous shock (for example, a fall in house prices) and amplified by the interplay between the shock and asset market dynamics. The propagating factor is leverage: when banks are highly leveraged, the initial shock and the ensuing reduction in asset prices induce massive asset liquidation, accentuating the price fall and possibly starting a vicious circle.

\begin{tabular}{clc} 
Parameters & Description & Set of Values \\
\hline$\phi^{*}$ & Target leverage & $\left\{\phi^{*} \in \mathbb{R}: 0<\phi^{*}<1\right\}$ \\
$\sigma$ & Variance of external assets & $\{\sigma \in \mathbb{R}: 0<\sigma<1\}$ \\
$\varepsilon$ & Bank reaction to the accounting rule & $\{\varepsilon \in \mathbb{R}: 0 \leq \varepsilon \leq 1\}$ \\
$\gamma$ & Price response of assets to banks' trades & $\{\gamma \in \mathbb{R}: \gamma>0\}$ \\
$h$ & Face value of interbank obligations & $\{h \in \mathbb{R}: h>0\}$ \\
$b$ & Face value of external funds & $\{b \in \mathbb{R}: b>0\}$ \\
$\beta$ & Sensitivity of interbank obligations to leverage & $\{\beta \in \mathbb{R}: 0<\beta<1\}$ \\
$r_{f}$ & Risk-free rate & $\left\{r_{f} \in \mathbb{R}: r_{f} \geq 0\right\}$ \\
\hline
\end{tabular}

Table 1: Range of values for each of the parameters in the model. For the sake of simplicity, we omitted the indices of banks and assets.

\section{Analysis}

The system of Eq. (11) represents a general framework based on which a number of exercises can be conducted and several issues can be investigated. In the present paper, we focus on a specific question that has recently risen to the top of the policy agenda:

RQ: "How does the risk of a systemic default depend on the interplay between the level of bank compliance with capital requirements and asset-market liquidity in the presence of a price shock?"

Toward this end, we first simplify the above modeling framework using mean-field approximation. Then, we provide a formal definition of systemic risk. Finally, we perform a numerical analysis of the probability of systemic default.

\subsection{Mean-field Approximation}

The theoretical literature shows that the exact propagation of shocks within the interbank market depends on the specific architecture of bank-to-bank financial linkages (see e.g., Allen and Gale, 2001; Freixas et al., 2000). However, the range of possible different architectures shrinks as network density increases. Therefore, when the network density is relatively high, the probability 


\section{ETH}

Eidgenössische Technische Hochschule Zürich Swiss Federal Institute of Technology Zurich

http://www.sg.ethz.ch
P. Tasca, S. Battiston:

Market Procyclicality and Systemic Risk

Submitted March 16, 2013

of systemic breakdown depends only weakly on the specific network architecture. Indeed, network density has been found to be a major driver of systemic risk (see e.g., Battiston et al., 2012a,b; Tasca and Battiston, 2011). There is also a body of empirical evidence that suggests that financial networks typically display a core-periphery structure with a dense core and a sparsely connected periphery (see e.g., Battiston et al., 2012c; Cont et al., 2011; Iori et al., 2006; Vitali et al., 2011). In the following, our aim is to describe the dynamics of the banks in the core of a core-periphery structure.

It has been argued that the financial sector has undergone increasing levels of homogeneity because of the spread of similar risk management models (e.g., VaR) and investment strategies - "[...]The level playing field resulted in everyone playing the same game at the same time, often with the same ball.", Haldane (2009). As a result, one might reason that, banks' balance sheet structures and portfolios tend to look alike. In particular, in this scenario, banks' leverage values may differ across banks and over time, but they will remain close to the average value.

Based on these considerations, we conduct a mean-field analysis of the model, attempting to study the behavior of the system in the neighborhood of a state in which the banks are close to homogeneous in terms of balance sheet management and interact with each other via the interbank network. Toward this end, we make the following three assumptions:

1. Banks set a similar reporting leverage targets, i.e., $\phi_{i}^{*} \approx \phi^{*}$.

2. There exists a simplified market in which the external assets are indistinguishable and uncorrelated and have identical initial values, i.e., $s_{1}(0)=s_{2}(0)=\ldots=s_{l}(0)$. In particular, this means that (i) all of the external assets have the same expected returns and the same volatility, (ii) the pairwise correlations are all zero (i.e., $\rho_{l k}=0, \forall l, k \in \Omega_{m}$ ), and (iii) the external assets have the same market impact (i.e., $\gamma_{l}=\gamma, \forall l \in \Omega_{m}$ ). The dynamics of the average price ${ }^{9}, s=\frac{1}{m} \sum_{l} s_{l}$, is $d s / s=\mu d t+\sigma d B$. In the absence of transaction costs, the $1 / \mathrm{m}$ heuristic is the optimal diversification strategy. Therefore, at time $t=0$, each bank $i$ composes an equally weighted portfolio of external assets, denoted as $p_{i}$, with $p_{i}=\sum_{l} Q_{i l} s_{l}=Q_{i} s$ where $Q_{i}=\sum_{l} Q_{i l}$ with $Q_{i 1}=Q_{i 2}=\ldots=Q_{i l}=\frac{Q_{i}}{m}$. Moreover, we assume that agents have comparable market power, i.e., that their portfolios of external assets are of a similar size, s.t. $Q_{i} \approx \frac{1}{n} \sum_{j} Q_{j}:=Q$. Therefore, $p_{i} \approx \frac{1}{n} \sum_{j} p_{j}:=p=Q s$. In addition, the agents are assumed to have the same level of compliance with capital requirements, i.e., $\varepsilon_{i}=\varepsilon, \forall i \in \Omega_{n}$.

3. The agents also have similar nominal total obligations in the interbank market, i.e., $h_{i} \approx$ $\frac{1}{n} \sum_{j} h_{j}:=h$, and similar total debt to external creditors, i.e., $b_{i} \approx \frac{1}{n} \sum_{j} b_{j}:=b$.

\footnotetext{
${ }^{9}$ Where the expected return is $\mu=\frac{1}{m} \sum_{l} \mu_{l}$ with $\mu_{1}=\mu_{2}=\ldots=\mu_{l}$, while $\sigma=\frac{\bar{\sigma}}{\sqrt{m}}$ with $\bar{\sigma}=\frac{1}{m} \sum_{l} \sigma_{l}$ and $B \sim \mathrm{N}(0, d t)$ is derived from the linear combination of $B_{1, \ldots, l, \ldots, m}$ uncorrelated processes.
} 


\section{ETH}

Eidgenössische Technische Hochschule Zürich Swiss Federal Institute of Technology Zurich

http://www.sg.ethz.ch
P. Tasca, S. Battiston:

Market Procyclicality and Systemic Risk

Submitted March 16, 2013

The first and the second assumptions imply Eq. (13), which reads the same for both individual trades of size $Q$ and the aggregate trade of size $n Q$. The second assumption also implies that the expected return on the average price is proportional to the relative change in the average trading size, i.e., $\mu=\gamma(d Q / Q)$, which therefore implies Eq. (12). The first and third assumptions imply that the mark-to-market value of interbank claims is similar across banks, i.e., $\hbar_{i}:=\sum_{j} W_{i j} \hbar_{j} \approx$ $\frac{1}{n} \sum_{j} \hbar_{j}:=\hbar=h /\left[1+r_{f}+\phi\right]^{-\hat{t}}$. The second and third assumptions imply that the ratio of external assets to total assets is homogeneous across banks, i.e., $\alpha_{i}=\sum_{l} \alpha_{i l}=p /(p+\hbar):=\alpha$. The third assumption implies that the ratio of external funds to total liabilities is homogeneous across banks, i.e., $\kappa_{i}=b /(b+h):=\kappa$. In totality, the three assumptions above imply that Eq. (5) simplifies to $\phi=(h+b) /\left(Q s+h\left[1+r_{f}+\beta \phi\right]^{-\hat{t}}\right)$, which is a quadratic expression in $\phi$. Without loss of generality, we set the risk-free rate equal to zero (i.e., $r_{f}=0$ ). We also set $\hat{t}=1$, which means that banks issue 1-year maturity obligations. Then, solving for $\phi$ and taking the positive root, we obtain Eq. (15).

In conclusion, under the above approximations and assumptions, the system of Eq. (11) simplifies to the following system of four coupled equations that will be studied in the remainder of our analysis

$$
\left\{\begin{aligned}
\frac{d s}{s} & =\gamma\left(\frac{d Q}{Q}\right) d t+\sigma d B \\
\frac{d Q}{Q} & =\left(\frac{\varepsilon}{\alpha}\right)\left(\frac{\phi^{*}-\phi}{1-\phi^{*}}\right) d t \\
\frac{d b}{b} & =\left(\frac{\varepsilon}{\kappa \phi}\right)\left(\frac{\phi^{*}-\phi}{1-\phi^{*}}\right) d t \\
\phi & =\frac{h(\beta-1)+\beta b-Q s+\left(4 \beta(b+h) Q s+(h-\beta(b+h)+Q s)^{2}\right)^{1 / 2}}{2 \beta Q s}
\end{aligned}\right.
$$

In this scenario, the balance sheet of each bank in the core can be represented as follows.

Balance-sheet

\begin{tabular}{|c|c|}
\hline Assets & Liabilities \\
\hline$\hbar$ & $h$ \\
$p$ & $b$ \\
& $e$ \\
\hline
\end{tabular}

\subsection{Systemic Risk}

We model the default event as a first passage time problem. An individual bank is assumed to default whenever the market value of its assets falls below the book value of its debts, even before their maturity. Accordingly, the default occurs whenever the bank's leverage is equal to or greater than one. 


\section{ETH}

Eidgenössische Technische Hochschule Zürich Swiss Federal Institute of Technology Zurich

http://www.sg.ethz.ch
P. Tasca, S. Battiston:

Market Procyclicality and Systemic Risk

Submitted March 16, 2013

However, the mean-field analysis enables us to assign a systemic meaning to the single bank default event. Indeed, banks manage overlapped portfolios composed of external assets and interbank claims. Consequently, banks are subject to similar sources of risk. Moreover, the leverage of each bank is close to the mean value across banks, i.e., $\phi_{i} \approx \phi, \forall i \in \Omega_{n}$. Hence, the failure of one bank $i$ in the core is likely to coincide with the failure of all of the other banks. This is also consistent with recent empirical studies (see e.g., Battiston et al., 2012c). We define systemic default as an event in which, at any time $t \geq 0$, the leverage $\phi$ is equal to or greater than one. Formally,

A systemic default occurs whenever $\phi(t) \geq 1$, given that $\forall t^{\prime}<t, \quad 0<\phi\left(t^{\prime}\right)<1$.

If the set $\Omega_{n}$ of banks is populated at a constant rate at each time interval $d t$, then the probability of systemic default $\mathbb{P}[$ systemic default $]$ is related to the expected default time $\bar{\tau}(\phi)$ and can be approximated as $\mathbb{P}$ [systemic default $] \approx d t / \bar{\tau}(\phi)$ with $d t<<\bar{\tau}(\phi) .{ }^{10}$ In the following, the probability of systemic default event is also referred to as systemic risk.

\subsection{Simulation Framework}

Before conducting the numerical experiments, we appropriately design the simulation framework with respect to the following: (1) the price shock, (2) the range of $\gamma$ and $\varepsilon$, and (3) the capital structure.

Aggregate shock. To assess the level of balance-sheet amplification, the exogenous aggregate price shock is assumed to have the following properties: (i) it is common, i.e., the shock is not assetspecific and cannot be diversified away; (ii) it is a single shock, i.e., it hits the external assets only once at the beginning of the simulation process; and (iii) it is uniform, i.e., the strength of the shock is homogeneous across assets.

Range of $\gamma$ and $\varepsilon$. It is convenient to generate a table $P_{(\varepsilon \times \gamma)}$ that represents the level of market procyclicality as a function of bank compliance with capital requirements $\varepsilon$ and asset-market impact $\gamma$. Accordingly, the market can be weakly, moderately or strongly procyclical (see Fig.2). For the former parameter, we choose a set of values that span the entire range of variation: $\varepsilon:=\{0.1,0.2, \ldots, 1\}$. Because our preliminary tests indicated that for values of $\gamma$ that are approximately larger than five, the results of the analysis do not change, so we choose the following set of values $\gamma:=\{0.1,0.2, \ldots, 5\}$.

Capital structure. First, note that under the assumption (2) used to derive the accounting rule in Eq. (6)-(7), $h(t)=h$ for all $t \geq 0$. Hereafter, for the sake of simplicity, we impose the initial condition $b+h=1$ and consider three different funding policies based on the imbalance towards

\footnotetext{
${ }^{10} \mathrm{~A}$ formal definition of $\bar{\tau}(\phi)$ is provided in Appendix B.
} 


\section{ETH}

Eidgenössische Technische Hochschule Zürich Swiss Federal Institute of Technology Zurich

http://www.sg.ethz.ch
P. Tasca, S. Battiston:

Market Procyclicality and Systemic Risk

Submitted March 16, 2013

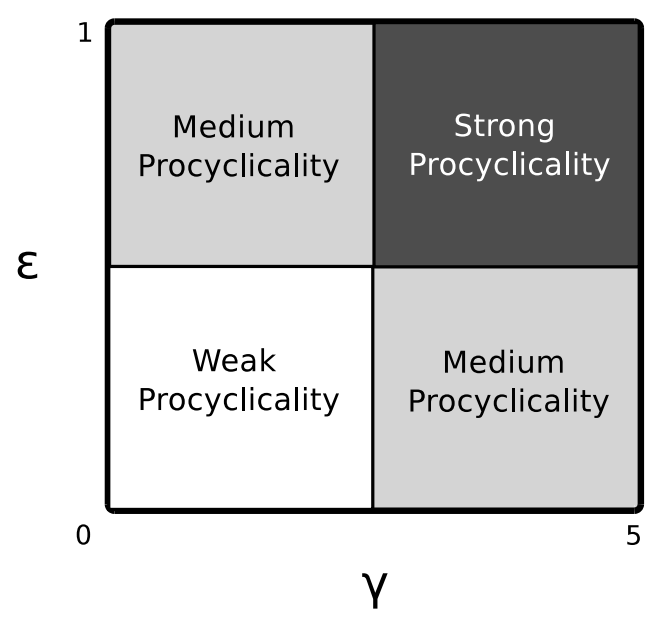

Figure 2: Table of Market Procyclicality. The left-bottom region of weak procyclicality represents a context in which the asset market is highly liquid (i.e., $\gamma \gtrsim 0$ ) and in which the banks loosely react to a asset-price changes (i.e., $\varepsilon \gtrsim 0$ ). The left-upper region of medium procyclicality represents a context in which the asset market is very liquid (i.e., $\gamma \gtrsim 0$ ) and in which the banks react promptly to asset-price changes (i.e., $\varepsilon \lesssim 1$ ). The right-bottom region of medium procyclicality represents a context in which the asset market is illiquid (i.e., $\gamma \lesssim 5$ ) and in which the banks loosely react to asset-price changes (i.e., $\varepsilon \gtrsim 0$ ). The right-upper region of strong procyclicality represents a context in which the asset market is highly illiquid (i.e., $\gamma \lesssim 5$ ) and in which the banks react promptly to asset-price changes (i.e., $\varepsilon \lesssim 1$ ).

external funds versus funds from the interbank market: high imbalance $(b=0.9>h=0.1)$, medium imbalance $(b=0.5 \equiv h=0.5)$, low imbalance $(b=0.1<h=0.9)$. We also consider three different levels of target leverage: low target $\left(\phi^{*}=0.7\right)$, medium target $\left(\phi^{*}=0.8\right)$, high target $\left(\phi^{*}=0.9\right)$. As a result, we have nine different capital structures, as illustrated in Tab. 2 . The remaining accounting entries for the balance sheets at time $t=0$ are obtained by solving the following system of equations:

$$
\begin{cases}p+\hbar=h+b+e & \text { (balance-sheet identity) } \\ \hbar=h /\left(1+\beta \phi^{*}\right) & \text { (market value of interbank claims) }\end{cases}
$$

with $p=Q s$. As $\beta \in(0,1)$, we use $\beta=0.5$ to represent a scenario in which interest rates on interbank claims have an intermediate level of sensitivity to the obligors' credit worthiness.

\subsection{Monte Carlo Simulations}

To compute the probability of systemic default, we first estimate the mean time to default $\bar{\tau}(\phi)$ based on the output of two thousand Monte Carlo simulations $(N=2.000)$ according to Eq. (12- 


\section{ETH}

Eidgenössische Technische Hochschule Zürich Swiss Federal Institute of Technology Zurich

http://www.sg.ethz.ch
P. Tasca, S. Battiston:

Market Procyclicality and Systemic Risk

Submitted March 16, 2013

15). ${ }^{11}$ Each realization lasts a number of steps $T=50.000$ with a step size of $\Delta t=0.01$. The mean time to default is obtained for all of the pairs $(\varepsilon, \gamma)$ in the table $P_{(\varepsilon, \gamma)}$ and all nine capital structures in Tab. 2. The leverage-price cycle, summarized by the flow-chart in Fig. 3, is the central part of our Monte Carlo analysis. In brief, at the beginning $(t=0)$, the leverage $\phi$ of the banks is set equal the target level $\phi^{*}$. To this value corresponds the "reference"price value $s^{*}:=\left[b+h+\phi^{*}(b \beta-h+\beta h)\right] /\left[\phi^{*} Q\left(1+\beta \phi^{*}\right)\right]$ as derived from Eq.(15). The initial price shock $(-10 \%)$ moves the banks out of "equilibrium" by deviating $s$ from $s^{*}$ and $\phi$ from $\phi^{*}$. To drive the leverage back toward $\phi^{*}$, banks adjust their external asset holdings and external funds according to the accounting rule in Eq. (13)-(14). The re-sizing of banks' balance sheets has a market impact on asset prices (see Eq. 12), which in turn further influence the balance sheet entries. This effect completes the leverage-asset price cycle (see Fig. 3). Systemic risk is then computed using the approximation in Section 3.2.

\section{Results}

Fig. 4-5-6 show the probability surface of the systemic default event as a function of (1) the level of market procyclicality and (2) the banks' capital structure. The capital structure, instead, refers to the balance between external and interbank funding sources. Finally, each figure corresponds to a different level of target leverage: $\phi^{*}=0.7,0.8,0.9$.

(1) Market Procyclicality In connection with the areas of the table $P_{(\varepsilon \times \gamma)}$ of market procyclicality in Fig. 2, the systemic risk in Fig. 4-5-6 varies as follows.

Weak procyclicality - left lower corner of $P_{(\varepsilon \times \gamma)} \quad$ Systemic risk is at its minimum level. Banks are in the region of weak market procyclicality characterized by a liquid asset market (i.e., $\gamma \gtrsim 0$ ) and a weak adjustment of any leverage deviation from the target level (i.e., $\varepsilon \gtrsim 0$ ). An example of sample path of leverage is illustrated in Fig. 7-c. Even though the price shock induces some asset liquidations, it is immediately absorbed by the system because of a sluggish balance-sheet management and a liquid asset market. Eventually, systemic risk remains at low levels.

Medium procyclicality - left upper corner of $P_{(\varepsilon \times \gamma)} \quad$ Systemic risk is at a moderate level. On the one side, banks promptly comply with capital requirements (i.e., $\varepsilon \lesssim 1$ ) and react to price shocks by selling orders of large size. Therefore, massive liquidation of banks' assets may put further downward pressure on asset prices. On the other side, the asset market is very liquid (i.e., $\gamma \gtrsim 0$ ). Therefore, potentially large quantities of the assets can be sold or bought without significant impact on their market price. Systemic risk remains at medium levels until

\footnotetext{
${ }^{11}$ The values of the parameters used in the simulations are reported in Tab. 3, and the procedure is described in greater detail in Appendix C.
} 


\section{ETH}

Eidgenössische Technische Hochschule Zürich Swiss Federal Institute of Technology Zurich

http://www.sg.ethz.ch
P. Tasca, S. Battiston:

Market Procyclicality and Systemic Risk

Submitted March 16, 2013

the liquidity does not "evaporate" from the market. An example of sample path of the leverage is illustrated in Fig. 7-a.

Medium procyclicality - right lower corner of $P_{(\varepsilon \times \gamma)}$ Systemic risk is at a moderate level. The asset-market is illiquid (i.e., $\gamma \lesssim 5$ ), and banks loosely comply with capital requirements (i.e., $\varepsilon \gtrsim 0)$. In this situation, the selling orders executed by the banks in response to the price shock are limited in size. Combined with a liquidity shock, these trades produce only a small price change, which is sufficient, however, to trigger a weak spiral of asset-price devaluation. This spiral further degenerates the banks' balance sheets and deteriorates the leverage. As a consequence, systemic risk slightly increases. An example of sample path of the leverage is illustrated in Fig. 7-d.

Strong procyclicality - right upper corner of $P_{(\varepsilon \times \gamma)} \quad$ Systemic risk is at its maximum level. Banks are in the region of strong market procyclicality characterized by the extremely dangerous combination of a highly illiquid asset market (i.e., $\gamma \lesssim 5$ ) and prompt responses to any deviation of the leverage from the target level (i.e., $\varepsilon \lesssim 1$ ). An example of sample path of the leverage is illustrated in Fig. 7-b. In this context, banks react to the price shock via selling orders of a significant size. If at the same time a liquidity shock materializes such that buyers temporarily retreat from the market, external assets can only be sold at "fire-sale prices". Eventually, the market crash degrades the balance sheets and both leverage and the default risk increase.

(2) Capital Structure Banks' capital structures also play an important role in systemic risk except under weak market procyclicality. Indeed, when banks' exposure to external funds is greater (i.e., $b>h$ ), a price shock will have a greater effect on banks' net worth, and systemic risk will be higher (as indicated by the light-gray surfaces in Fig. 4-5-6). In contrast, when banks' positions are more tilted towards the interbank market, the price shock is damped down because it mainly propagates via a drop in the market value of the interbank claims (as indicated by the dark-gray surfaces in Fig. 4-5-6). In this case, the systemic risk is lower. Interestingly, the gaps between the levels of systemic risk associated with the three different funding policies are amplified at high levels of market procyclicality (see the border between the surfaces in Fig. 45-6). Finally, the higher the target leverage level is, the more pronounced the effects (compare Fig. 4-5-6). Indeed, leverage is the propagating factor of a price shock: if banks did not target leverage, letting equity absorb the shock, the vicious circle would be mitigated or even eliminated.

Overall, the results suggest that to mitigate the systemic effects of an unexpected price shock, two complementary policy rules can be implemented: (1) one can allow banks to weaken their compliance in adjusting their leverage to meet the target level (i.e., $\varepsilon$ will decrease toward zero); ${ }^{12}$ or (2) one can set up financing facilities to increase market liquidity (i.e., $\gamma$ will decrease toward

\footnotetext{
${ }^{12}$ Based on our Monte Carlo trials, we find that the leverage follows a slower mean reversion process toward the target level. Interestingly, this effect could explain the empirical puzzle recently discovered by Adrian and Shin (2008b) concerning the slow mean reversion over time exhibited by the (reported) leverage ratios.
} 


\section{ETH}

Eidgenössische Technische Hochschule Zürich Swiss Federal Institute of Technology Zurich

http://www.sg.ethz.ch
P. Tasca, S. Battiston:

Market Procyclicality and Systemic Risk

Submitted March 16, 2013

zero). If one (both) of these two policy rules is put in place, the market will transition into the region of medium (weak) procyclicality. If, moreover, one can reduce banks' imbalance towards the external market and set a cap to the target leverage, the systemic risk will further decrease. ${ }^{13}$ Indeed, for containing macro-prudential risks, leverage caps could be helpful if other solutions based on capital or contingent rules turn out to be too costly or difficult to implement. However, one drawback of this rule lies in its difficulty to be harmonized among different business models and cross-border banks. Moreover, leverage caps should be robust to: (i) the accounting treatment of off-balance operations (incl., derivatives and hedges); and (ii) the treatment of leverage embedded in structured finance products.

\section{Concluding Remarks}

In this paper, we combined a balance sheet approach with a dynamic stochastic setting to investigate the impact of market procyclicality on systemic risk. One of the novelties of our work is that it provides a quantitative representation of the notion of market procyclicality using a table whose dimensions correspond to (1) the level of bank compliance with capital requirements (controlled by the parameter $\varepsilon$ ) and (2) the degree of asset market liquidity (controlled by the parameter $\gamma$ ).

The general approach is to perturb the system with an aggregate price shock and analyze the probability of systemic default in critical regions of the table of market procyclicality. In our model, systemic defaults are a result of drops in asset prices, which are endogenously driven by bank trades. Thus, the leverage-asset price cycle is characterized by a "self-fulfilling" dynamics. Essentially, over-leveraged banks (which are more likely to default) tend to liquidate their assets after negative price variations. However, their market impact further decreases asset prices. Thus, balance-sheet management may amplify the effect of an initial shock, turning it into a spiral of asset price devaluation. Hence, at the heart of our model are pecuniary externalities in the form of direct asset price contagion (via overlapping portfolios) and indirect asset price contagion (via interbank claims). In particular, we stress the policy implications of the interplay between bank capital requirements and asset market liquidity. When the asset market is very liquid, even a strong compliance with capital requirements, which are usually alleged to be procyclical, do not in fact increase the probability of systemic default. Conversely, when the asset market is very illiquid, even a weak compliance with capital requirements increase the probability of systemic default.

\footnotetext{
${ }^{13}$ The G20 group of leading countries agreed on September 2012 to introduce a leverage ratio on banks by the end of 2012, as part of the Basel III reform from the global Basel Committee on Banking Supervision, to make the sector less risky.
} 


\section{ETH}

Eidgenössische Technische Hochschule Zürich Swiss Federal Institute of Technology Zurich

http://www.sg.ethz.ch
P. Tasca, S. Battiston:

Market Procyclicality and Systemic Risk

Submitted March 16, 2013

Overall, this paper sheds light on the tension between (1) the individual incentive to aim for a particular ratio of $\mathrm{VaR}$ to economic capital and reduce idiosyncratic shocks through asset diversification and (2) the homogenized system that results from the fact that banks adopt the same business and risk management practices. From an individual firm perspective, the application of the accounting rule and asset diversification is a sensible strategy. It is important to note that every bank is acting perfectly rationally from its own individual perspective. However, from a systemic perspective, this strategy generates an undesirable result. In fact, when banks start moving in a "synchronized" manner, they may amplify the effects of even small external shocks on assets dispersed across banks.

One possible illustrative application of our modeling framework is the U.S. sub-prime crisis (2007-2009). In that crisis, the eternal asset market would be the market for mortgage-backed securities, which was hit by a fundamental shock (i.e., the collapse of the U.S. housing bubble). As in our model, many of the largest financial institutions exposed to those assets were highly target-leveraged, were interconnected via the interbank market and made use of mark-to-market accounting. At the peak of the crisis, banks stopped lending to each other, and the market froze. To reduce the risk of systemic collapse, the Federal Reserve injected additional liquidity and helped banks to reduce their exposure to the external asset market by buying a portion of the mortgage-backed securities. Therefore, our analysis suggests that policy makers should employ macro-prudential supervisory risk assessment policies in coordination with monetary policies to compensate for the effect of market-wide liquidity in the presence of aggregate shocks.

The simplicity of our theoretical framework allows for the present research to be extended in several ways. For instance, the core of banks could be understood as "too-big-to-fail", and one could try to model the moral hazard problem ${ }^{14}$, which could induce excessive risk-taking with regard to external assets. Researchers might also consider the effect of the heterogeneity of banks' balance-sheet structures and target leverage levels as well as heterogeneity in terms of asset price volatility and drift. Finally, one could include dynamic link formation both in the interbank network and in the network of banks and external assets.

\footnotetext{
${ }^{14}$ Moral hazard would arise from banks' expectations concerning the intervention of the central bank in providing liquidity or reducing exposure to external assets.
} 


\section{ETH}

Eidgenössische Technische Hochschule Zürich Swiss Federal Institute of Technology Zurich

http://www.sg.ethz.ch
P. Tasca, S. Battiston:

Market Procyclicality and Systemic Risk

Submitted March 16, 2013

\begin{tabular}{|c|c|}
\hline \multicolumn{2}{|c|}{$\phi^{*}=0.8$} \\
\hline Assets & Liabilities \\
\hline$\hbar=0.071$ & $h=0.1$ \\
\hline \multirow{2}{*}{$p=1.179$} & $b=0.9$ \\
\hline & $e=0.25$ \\
\hline Assets & Liabilities \\
\hline$\hbar=0.357$ & $h=0.5$ \\
\hline \multirow[t]{2}{*}{$p=0.893$} & $b=0.5$ \\
\hline & $e=0.25$ \\
\hline Assets & Liabilities \\
\hline$\hbar=0.643$ & $h=0.9$ \\
\hline \multirow[t]{2}{*}{$p=0.607$} & $b=0.1$ \\
\hline & $e=0.25$ \\
\hline
\end{tabular}

\begin{tabular}{|c|c|}
\hline \multicolumn{2}{|c|}{$\phi^{*}=0.9$} \\
\hline Assets & Liabilities \\
\hline$\hbar=0.069$ & $h=0.1$ \\
\hline \multirow[t]{2}{*}{$p=1.041$} & $b=0.9$ \\
\hline & $e=0.11$ \\
\hline Assets & Liabilities \\
\hline$\hbar=0.345$ & $h=0.5$ \\
\hline \multirow[t]{2}{*}{$p=0.765$} & $b=0.5$ \\
\hline & $e=0.11$ \\
\hline Assets & Liabilities \\
\hline$\hbar=0.620$ & $h=0.9$ \\
\hline \multirow[t]{2}{*}{$p=0.490$} & $b=0.1$ \\
\hline & $e=0.11$ \\
\hline
\end{tabular}

Table 2: Banks' capital structures at time $t=0$ as used in the Monte Carlo simulations, based on three different funding policies and three different levels of target leverage.

\begin{tabular}{l|l|l} 
Model Parameters & Simulation Setting & Initial Conditions \\
\hline$\sigma=0.1$ & shock $=-10 \%$ & $b_{0}=0.1,0.5,0.9$ \\
$r_{f}=0$ & $N=2.000$ & $Q_{0}=1$ \\
$\beta=0.5$ & $\Delta t=0.01$ & $s_{0}=s^{*} \times($ shock +1$)$ \\
$\varepsilon=0.1: 0.1: 1$ & $T=50.000$ & \\
$\gamma=0.1: 0.1: 5$ & & \\
$h=0.1,0.5,0.9$ & & \\
\hline
\end{tabular}

Table 3: Range of values and initial conditions used in the simulation analysis. 


\section{EIH}

Eidgenössische Technische Hochschule Zürich Swiss Federal Institute of Technology Zurich

http://www.sg.ethz.ch
P. Tasca, S. Battiston:

Market Procyclicality and Systemic Risk

Submitted March 16, 2013

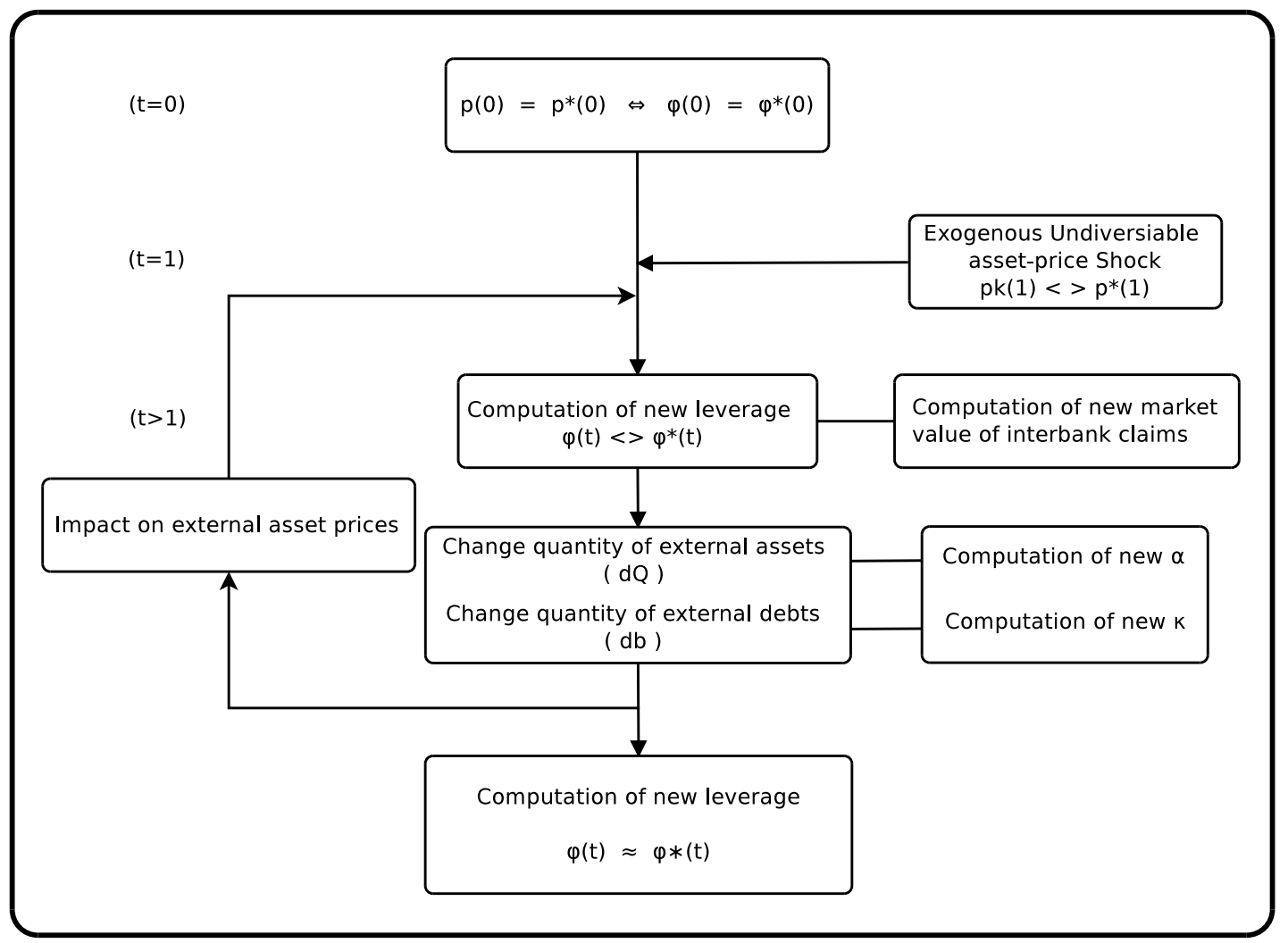

Figure 3: Flow-chart of the numerical simulation of the financial system in Eq. (12-15) with an emphasis on the leverage-price cycle. Note that we use $p_{k}$ to denote the new portfolio value after the price shock. 


\section{EIH}

Eidgenössische Technische Hochschule Zürich Swiss Federal Institute of Technology Zurich

http://www.sg.ethz.ch
P. Tasca, S. Battiston:

Market Procyclicality and Systemic Risk

Submitted March 16, 2013

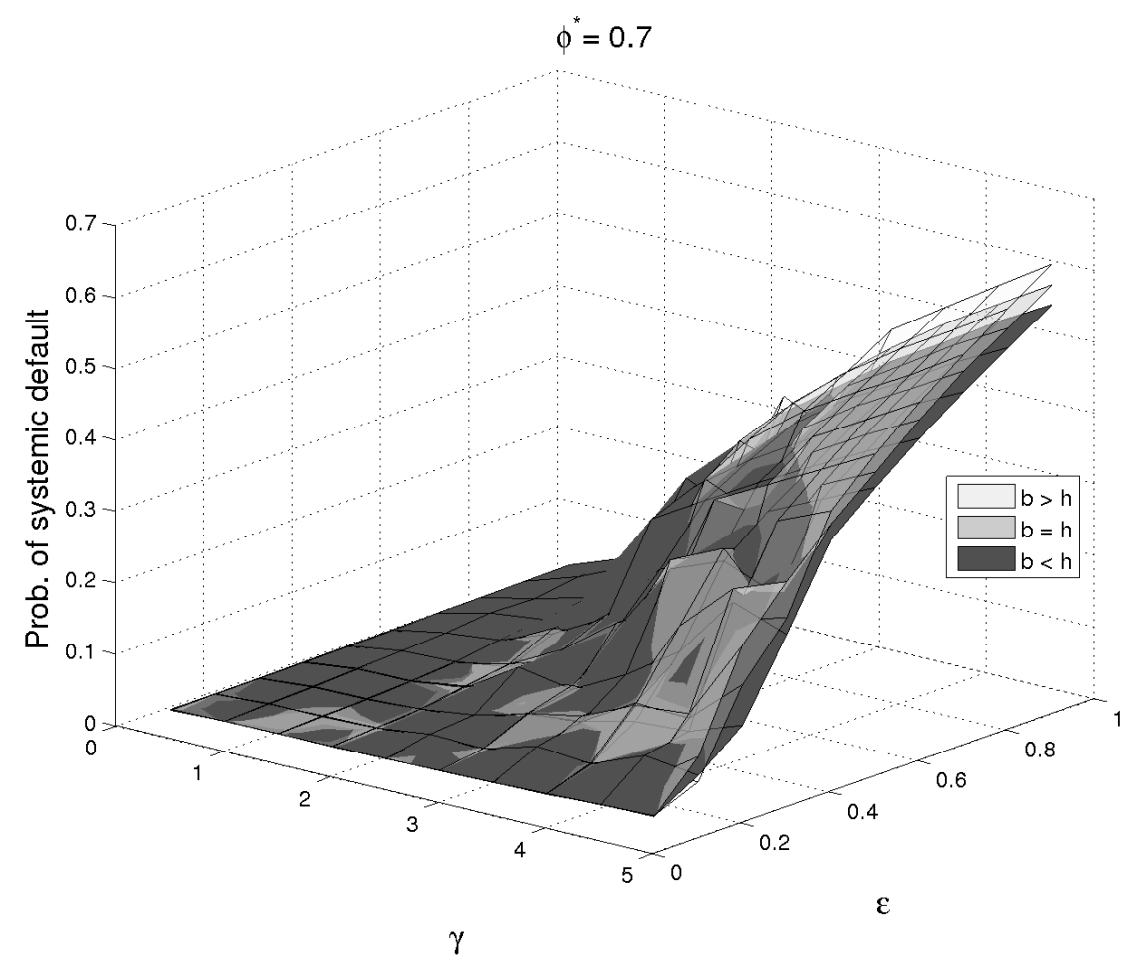

Figure 4: Surface probability of systemic default when banks' target leverage is 0.7. In the x-axis, $\gamma$ represents the average price response to trades. In the y-axis, $\varepsilon$ represents the intensity of bank compliance with capital requirements. The light gray surface shows the probability of systemic default when banks have high imbalance towards external funds $(b>h)$. The gray surface shows the probability of systemic default when banks have medium imbalance towards external funds $(b=h)$. The dark gray surface shows the probability of systemic default when banks have high imbalance towards external funds $(b>h)$. 


\section{EIH}

Eidgenössische Technische Hochschule Zürich Swiss Federal Institute of Technology Zurich

http://www.sg.ethz.ch
P. Tasca, S. Battiston:

Market Procyclicality and Systemic Risk

Submitted March 16, 2013

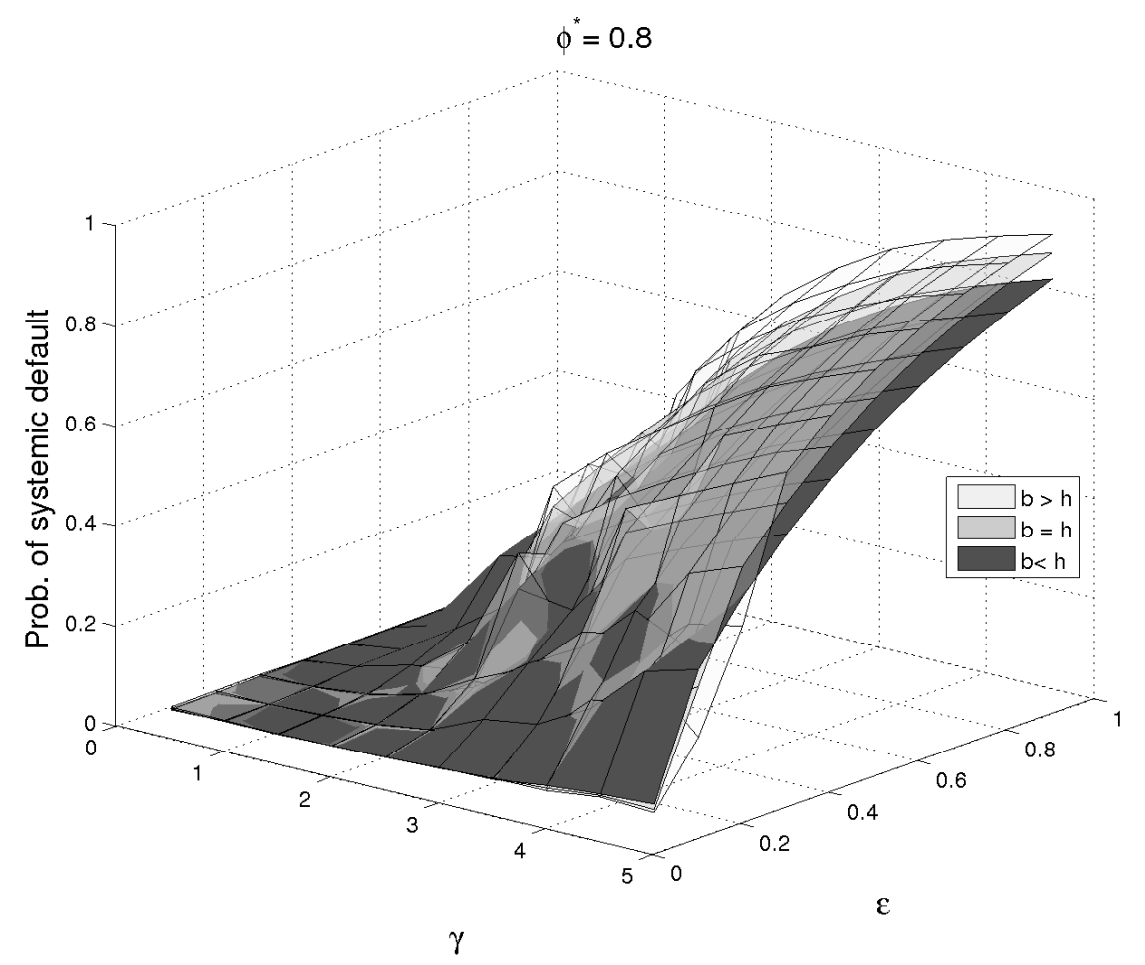

Figure 5: Surface probability of systemic default when banks' target leverage is 0.8 .

For a description of the surfaces see Fig. 4. 


\section{EIH}

Eidgenössische Technische Hochschule Zürich Swiss Federal Institute of Technology Zurich

http://www.sg.ethz.ch
P. Tasca, S. Battiston:

Market Procyclicality and Systemic Risk

Submitted March 16, 2013

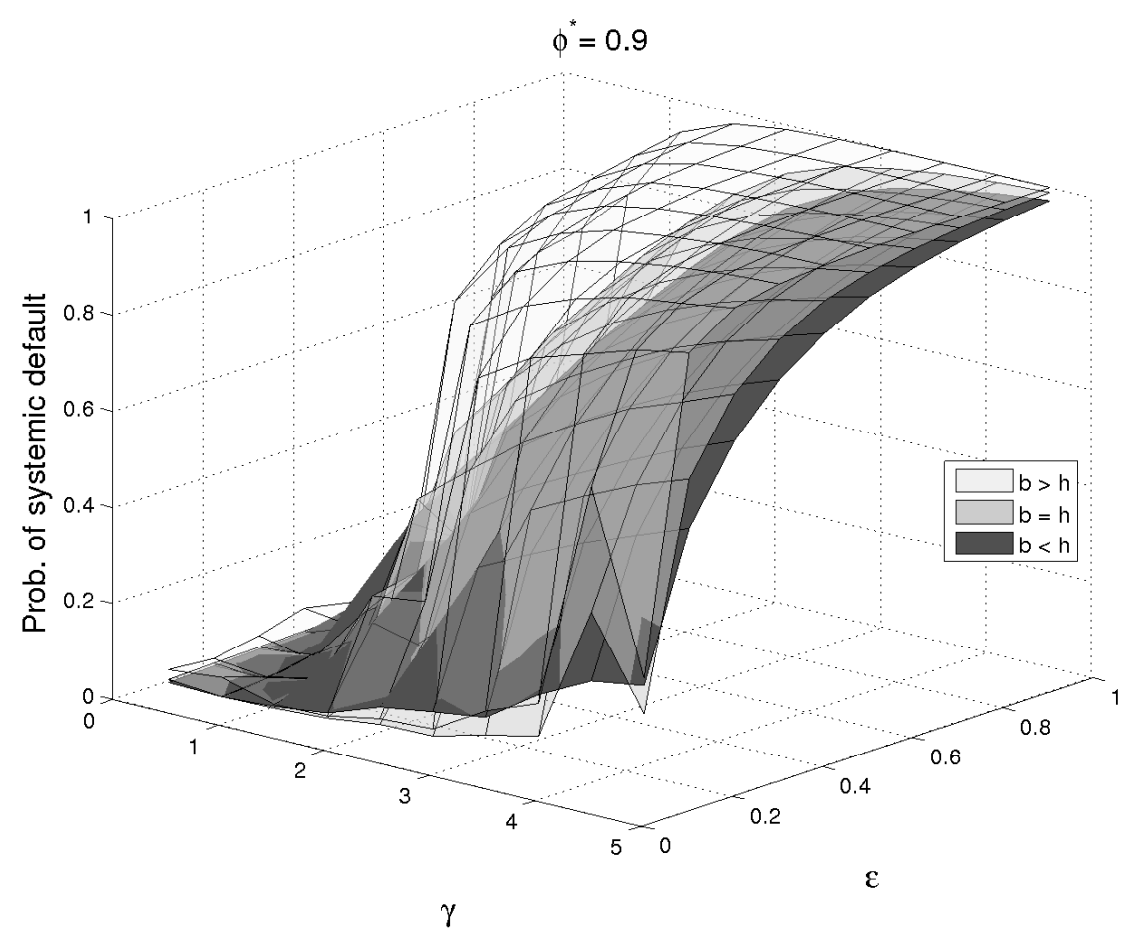

Figure 6: Surface probability of systemic default when banks' target leverage is 0.9 .

For a description of the surfaces see Fig. 4. 


\section{EIH}

Eidgenössische Technische Hochschule Zürich Swiss Federal Institute of Technology Zurich

http://www.sg.ethz.ch
P. Tasca, S. Battiston:

Market Procyclicality and Systemic Risk

Submitted March 16, 2013

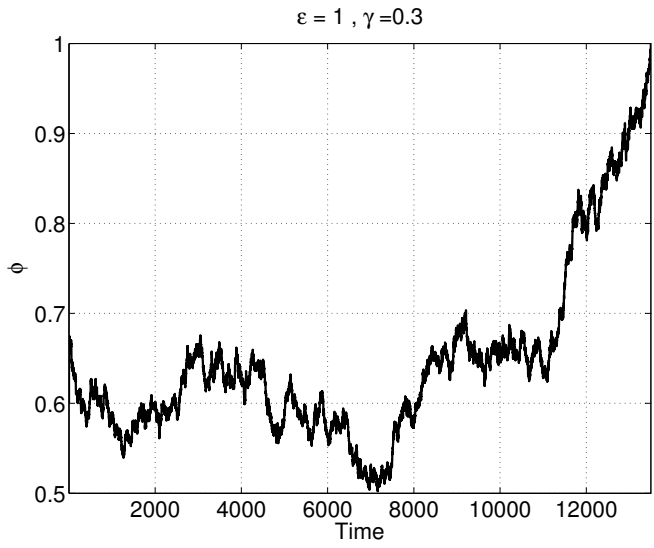

(a)

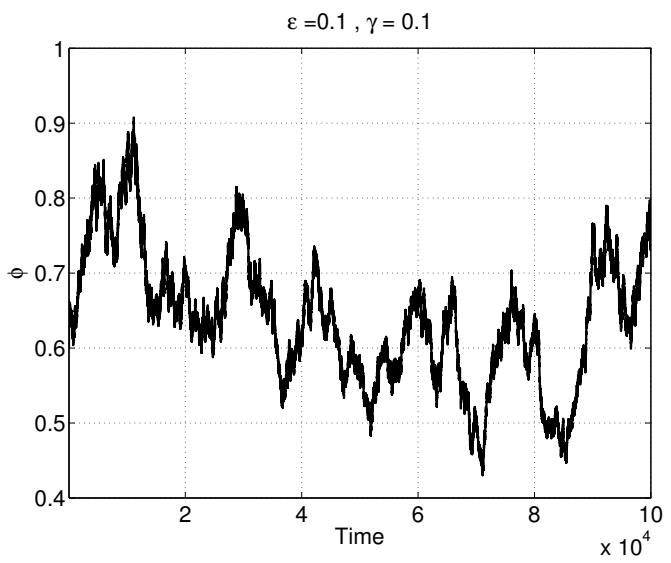

(c)

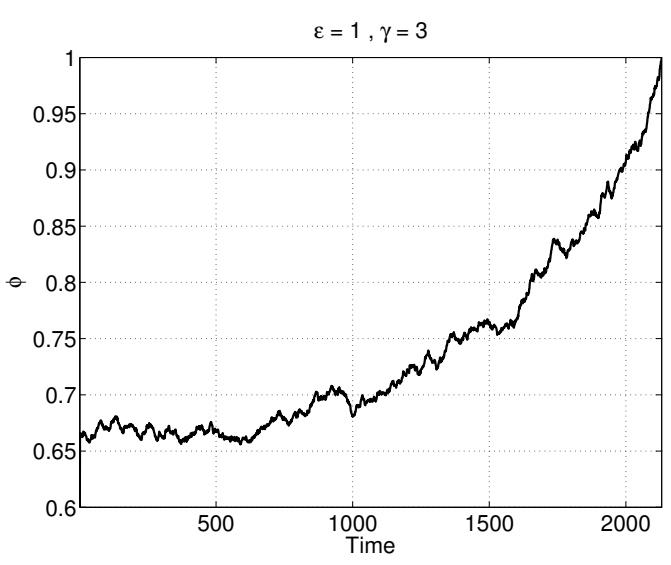

(b)

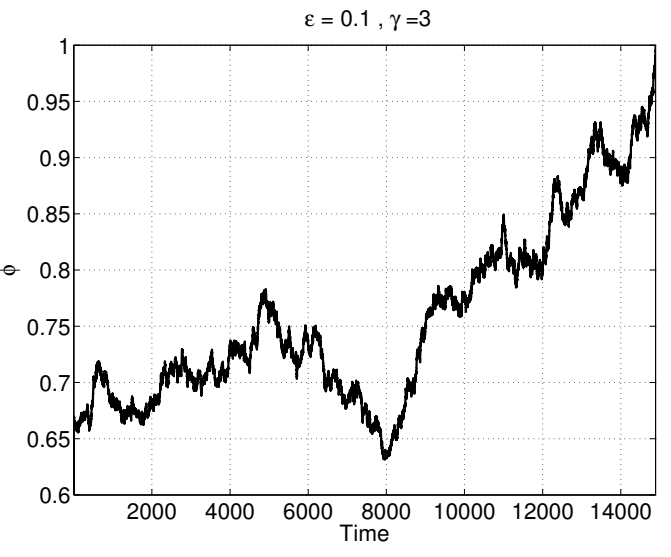

(d)

Figure 7: Sample paths for the leverage with target leverage $\phi^{*}=0.6$. The target leverage works as a constant long-run mean. However, the leverage follows a process that is non-stationary. Indeed, the leverage dynamics is derived by the non-linear system in Section 3.1 in which the price process in Eq.12 is non-stationary. Nevertheless, in case (c) of weak procyclicality in which both $\varepsilon$ and $\gamma$ approximate zero, the path resembles a stationary process with mean reversion level equal to 0.6. In this case, the dynamics of: (1) the price process in Eq.12; (2) the bank asset trading in Eq.13, and (3) the external funds in Eq.14 are damped down by the parameter $\varepsilon \equiv \gamma=0.1$. 


\section{ETH}

Eidgenössische Technische Hochschule Zürich Swiss Federal Institute of Technology Zurich

http://www.sg.ethz.ch
P. Tasca, S. Battiston:

Market Procyclicality and Systemic Risk

Submitted March 16, 2013

\section{A An Accounting Rule Based on Target Leverage}

In this section we derive the accounting rule in Eq. (6-7), according to which a generic bank $i \in \Omega_{n}$ adjusts its balance sheet entries in response to an asset price change. At time $\mathrm{t}=0$, bank $i$ starts its activity with a target leverage, $\phi_{i}(0) \equiv \phi_{i}^{*}$. As pointed out in Section 2.3, the accounting rule is based on the underlying assumption that bank $i$ keeps its debt/credit positions fixed in the interbank market: the bank only adjusts the quantity of external assets and external funds: $h_{i}(0)=h_{i}$ and $\sum_{j} W_{i j}(0) \hbar_{j}(0)=\sum_{j} W_{i j} \hbar_{j}$ for all $t \geq 0$ and all $i, j \in \Omega_{n}$. Thus, the initial leverage can be written as $\phi_{i}(0)=\frac{h_{i}+b_{i}(0)}{\sum_{l} Q_{i l}(0) s_{l}(0)+\sum_{j} W_{i j} \hbar_{j}}$. At time $\mathrm{t}=1$, for the sake of simplicity, let only one external asset $l \in \Omega_{m}$ be shocked. Ceteris paribus (i.e., $b_{i}$ and $Q_{i l}$ remain as they were at time $\mathrm{t}=0$ ), the new price value $s_{l}(1)$ implies the following leverage: $\phi_{i}(1)=\frac{h_{i}+b_{i}(0)}{\sum_{l} Q_{i l}(0) s_{l}(1)+\sum_{j} W_{i j} \hbar_{j}} \neq \phi_{i}^{*}$. After the price shock, at time $\mathrm{t}=2$, the bank is able to adjust its leverage to the target level by changing the quantity of external asset $l$ held on the asset side - i.e., $\sum_{l} Q_{i l}(0) \rightarrow \sum_{l} Q_{i l}(2)$, and of the external debts on the liability side - i.e., $b_{i}(0) \rightarrow b_{i}(2)$. As a result, the new leverage at time $\mathrm{t}=2$ is equal to the target level, i.e., $\phi_{i}(2) \equiv \phi_{i}^{*}$. Expanding the equivalence $\phi_{i}(2) \equiv \phi_{i}(0) \equiv \phi_{i}^{*}$ yields

$$
\phi_{i}(2)=\frac{h_{i}+b_{i}(2)}{\sum_{l} Q_{i l}(2) s_{l}(1)+\sum_{j} W_{i j} \hbar_{j}} \equiv \frac{h_{i}+b_{i}(0)}{\sum_{l} Q_{i l}(0) s_{l}(0)+\sum_{j} W_{i j} \hbar_{j}} \equiv \phi_{i}^{*} .
$$

Now, the equation for leverage at time $\mathrm{t}=1$ can be re-written as $\sum_{j} W_{i j} \hbar_{j}+\sum_{l} Q_{i l}(0) s_{l}(1)=\frac{h_{i}+b_{i}(0)}{\phi_{i}(1)} \Rightarrow$ $\sum_{j} W_{i j} \hbar_{j}=\frac{h_{i}+b_{i}(0)}{\phi_{i}(1)}-\sum_{l} Q_{i l}(0) s_{l}(1)$, which we substitute into the equation for the leverage at time $\mathrm{t}=2$ as follows:

$$
\phi_{i}(2)=\frac{h_{i}+b_{i}(2)}{\sum_{l} Q_{i l}(2) s_{l}(1)+\frac{h_{i}+b_{i}(0)}{\phi_{i}(1)}-\sum_{l} Q_{i l}(0) s_{l}(1)}=\frac{h_{i}+b_{i}(2)}{\frac{h_{i}+b_{i}(0)}{\phi_{i}(1)}+\Delta Q_{i l}(2) s_{l}(1)},
$$

where $\Delta Q_{i l}$ indicates the variation in the quantity of external assets in bank $i$ 's portfolio due to a change in its holdings of external asset $l$. Eq. (17) can be re-written as

$$
\frac{h_{i}+b_{i}(0)}{\phi_{i}(1)}+\Delta Q_{i l}(2) s_{l}(1) \equiv \frac{h_{i}+b_{i}(2)}{\phi_{i}^{*}}
$$

Based on the assumptions associated with the accounting rule as indicated in Section 2.3, the following identity holds true:

$$
b_{i}(2)=b_{i}(0)+\Delta Q_{i l}(2) s_{l}(1) .
$$

Then, substituting (19) into (18) yields $\frac{h_{i}+b_{i}(0)}{\phi_{i}(1)}+\Delta Q_{i l}(2) s_{l}(1)=\frac{h_{i}+b_{i}(0)+\Delta Q_{i l}(2) s_{l}(1)}{\phi_{i}^{*}}$, from which, with little arrangements we obtain

$$
\Delta Q_{i l}(2) s_{l}(1)=\frac{h_{i}+b_{i}(0)}{\phi_{i}(1)}\left(\frac{\phi_{i}^{*}-\phi_{i}(0)}{1-\phi_{i}^{*}}\right) .
$$

Therefore, the rate of change in the demand for asset $l$ from bank $i$ is:

$$
\frac{Q_{i l}(2)-Q_{i l}(0)}{Q_{i l}(0)}=\frac{\sum_{j} W_{i j} \hbar_{i j}+Q_{i l}(0) s_{l}(1)}{Q_{i l}(0) s_{l}(1)}\left(\frac{\phi_{i}^{*}-\phi_{i}(0)}{1-\phi_{i}^{*}}\right)=\frac{1}{\alpha_{i l}}\left(\frac{\phi_{i}^{*}-\phi_{i}(0)}{1-\phi_{i}^{*}}\right),
$$




\section{ETH}

Eidgenössische Technische Hochschule Zürich Swiss Federal Institute of Technology Zurich

http://www.sg.ethz.ch
P. Tasca, S. Battiston:

Market Procyclicality and Systemic Risk

Submitted March 16, 2013

where $\alpha_{i l}=\frac{Q_{i l}(0) s_{l}(1)}{a_{i}(1)}$ is the ratio of the value of the external asset $l$ to the total asset value held by the bank. The relative change in debts is easily obtained from Eq. 19:

$$
\frac{b_{i}(2)-b_{i}(0)}{b_{i}(0)}=\frac{h_{i}+b_{i}(0)}{b_{i}(0) \phi_{i}(0)}\left(\frac{\phi_{i}^{*}-\phi_{i}(0)}{1-\phi_{i}^{*}}\right)=\frac{1}{\kappa_{i} \phi_{i}(0)}\left(\frac{\phi_{i}^{*}-\phi_{i}(0)}{1-\phi_{i}^{*}}\right),
$$

where $\kappa_{i}=\frac{b_{i}(0)}{h_{i}+b_{i}(0)}$ is the ratio of external debts to the total debts. In the presence of market frictions, ${ }^{15}$ the bank may react with a certain (in)elasticity $\varepsilon_{i} \in(0,1]$ to deviations in leverage $\phi_{i}$ from the target level. Heuristically, in continuous time, the accounting rule implies the following (rates of) change, respectively, for the demand for external assets and the amount of external debts:

$$
\begin{gathered}
\frac{d Q_{i l}}{Q_{i l}}=\left(\frac{\varepsilon_{i}}{\alpha_{i l}}\right)\left(\frac{\phi_{i}^{*}-\phi_{i}}{1-\phi_{i}^{*}}\right) d t \\
\frac{d h_{i}}{h_{i}}=\left(\frac{\varepsilon_{i}}{\kappa_{i} \phi_{i}}\right)\left(\frac{\phi_{i}^{*}-\phi_{i}}{1-\phi_{i}^{*}}\right) d t .
\end{gathered}
$$

The boundary conditions are: (i) $\frac{d Q_{i}}{Q_{i}}=\sum_{l}\left(\frac{d Q_{i l}}{Q_{i l}}\right)$ s.t. $\frac{d Q_{i l}}{Q_{i l}}=0$ for some $l \in \Omega_{m}$; (ii) $d Q_{i l} \geq-Q_{i l}$ for all $l \in \Omega_{m}$ and $i \in \Omega_{n}$, and (iii) $d h_{i} \geq-h_{i}$ for all $i \in \Omega_{n}$. The accounting rule in Eq. (23) can be easily generalized if bank $i$ changes the quantity of more than one external asset. For example, the rule for changing the quantity of all of the assets in $\Omega_{m}$ is

$$
\frac{d Q_{i}}{Q_{i}}=\left(\frac{\varepsilon_{i}}{\alpha_{i}}\right)\left(\frac{\phi_{i}^{*}-\phi_{i}}{1-\phi_{i}^{*}}\right) d t
$$

where $Q_{i}=\sum_{l} Q_{i l}$ and $\alpha_{i}=\sum_{l} \alpha_{i l}$.

\section{B Expected time-to-default}

The first time to default $\tau(\phi)$, is defined as the first time the process $\{\phi\}_{t \geq 0}$ touches the upper bound of the set $\Omega_{\phi}:=(0,1]$. Namely, $\tau(\phi)=\inf \{t \geq 0: \phi(t) \geq 1\}$ with $\inf \{\emptyset\}=T$ if one is never reached. The full characterization of $\tau(\phi)$ is its transition probability density function $f_{\phi}\left(1, t \mid \phi_{0}, 0\right)$ conditional to the initial value $\phi(0):=\phi_{0} \in(0,1)$. Then, the mean time to default is

$$
\bar{\tau}(\phi):=\int_{0}^{T} t f_{\phi}\left(1, t \mid \phi_{0}, 0\right) d t
$$

which is the mean first hitting time for $\{\phi\}_{t \geq 0}$ to reach the absorbing default barrier before the terminal date $T$.

\footnotetext{
${ }^{15}$ I.e., market and (or) firm structures that prevent banks from instantly adjusting their balance sheets using the accounting rule.
} 


\section{ETH}

Eidgenössische Technische Hochschule Zürich Swiss Federal Institute of Technology Zurich

http://www.sg.ethz.ch
P. Tasca, S. Battiston:

Market Procyclicality and Systemic Risk

Submitted March 16, 2013

\section{Numerical Analysis}

In this section, we show how to obtain the mean time to default from the system (11), through simulations of the SDEs. The first step is to simulate the standard Brownian motion. Then, we look at stochastic differential equations, after which we compute the exit time for the process $\{\phi\}_{t \geq 0}$ through the upper barrier fixed at one.

Simulation of Brownian Motion We begin by considering a discretized version of Brownian motion. We set the step-size as $\Delta t$ and let $B_{\ell}$ denote $B\left(t_{\ell}\right)$ with $t_{\ell}=\ell \Delta t$. According to the properties of Brownian motion, we find

$$
B_{\ell}=B_{\ell-1}+d B_{j} \quad \ell=1, \ldots, L .
$$

Here, $L$ denotes the number of steps that we take with $t_{0}, t_{1}, \ldots, t_{L}$ as a discretization of the interval $[0, T]$, and $d B_{\ell}$ is a normally distributed random variable with zero mean and variance $\Delta t$. Expression (27) can be seen as a numerical recipe to simulate Brownian motion.

Simulation of the SDEs We use the Euler-Maruyama method. First, we discretize the dynamics of the processes $\{s\}_{t \geq 0},\{Q\}_{t \geq 0}$ and $\{b\}_{t \geq 0}$. Then, we substitute the value of $s, b$ and $Q$ into the discrete time version of Eq.(15). It is unnecessary to derive the dynamics of $\{\phi\}_{t \geq 0}$ via Ito's Lemma from the dynamics of $\{s\}_{t \geq 0},\{Q\}_{t \geq 0}$ and $\{b\}_{t \geq 0}$. We directly use the expression in Eq.(15), which is the closedform solution for $\phi$ in terms of $s, Q$ and $b$. Therefore, the discrete version of the system of equations in Section 3.1 reads as follows:

$$
\left\{\begin{aligned}
s_{\ell} & =s_{\ell-1}+\mu_{s}\left(\phi_{\ell-1}, s_{\ell-1}, \phi^{*}, \gamma, \varepsilon, \alpha_{\ell}\right) \Delta t+\sigma\left(s_{\ell-1}\right) d B_{\ell}, \quad s(0)=s_{0} \\
Q_{\ell} & =Q_{\ell-1}+\mu_{Q}\left(\phi_{\ell-1}, \phi^{*}, \varepsilon, \alpha_{\ell}\right) \Delta t, \quad Q(0)=Q_{0} \\
b_{\ell} & =b_{\ell-1}+\mu_{b}\left(\phi_{\ell-1}, b_{\ell-1}, \phi^{*}, \varepsilon, \kappa_{\ell}\right) \Delta t, \quad b(0)=b_{0} \\
\phi_{\ell} & =\frac{h(\beta-1)+\beta b_{\ell}-Q_{\ell} s_{\ell}+\sqrt{4 \beta\left(b_{\ell}+h\right) Q_{\ell} s_{\ell}+\left(h-\beta\left(b_{\ell}+h\right)+Q_{\ell} s_{\ell}\right)^{2}}}{2 \beta Q_{\ell} s_{\ell}}
\end{aligned}\right.
$$

where $\alpha_{\ell}=\left(Q_{\ell-1} s_{\ell-1}\right) /\left(Q_{\ell-1} s_{\ell-1}+\hbar_{\ell}\right), \hbar_{\ell}=h /\left(1+\beta \phi_{\ell-1}\right), \kappa_{\ell}=b_{\ell-1} /\left(b_{\ell-1}+h\right)$. Here $\phi_{\ell}, s_{\ell}, b_{\ell}$, $\alpha_{\ell}, \hbar_{\ell}, Q_{\ell}$ and $\kappa_{\ell}$ are the approximation to $\phi(\ell \Delta t), s(\ell \Delta t), b(\ell \Delta t), \alpha(\ell \Delta t), \hbar(\ell \Delta t), Q(\ell \Delta t)$ and $\kappa(\ell \Delta t)$. While, $\Delta t$ is the step size, $d B_{\ell}=B_{\ell}-B_{\ell-1}$ and $L$ is the number of steps we take.

Estimation of expected time to default The expected exit time can be estimated in the following manner. We simulate a single sample path for $\{s\}_{t \geq 0},\{Q\}_{t \geq 0},\{b\}_{t \geq 0}$ and compute the value $\phi_{\ell}$. Then, we observe the time at which the process $\{\phi\}_{t \geq 0}$ crossed the default boundary fixed at one. Once this point has been reached, we halt the simulation. The observed time approximates the exit time in the current sample path. If the trajectory of $\{\phi\}_{t \geq 0}$ does not exit through one before $T$, we set the exit time equal to $T$, i.e., the economy terminal date. By using the Monte Carlo method, we estimate the expected exit time for the system by simulating a total of $N$ sample paths within $[0, T]$. We keep track of the fraction of trajectories that did not cross the default boundary before $T$ and make sure to set $T$ to keep this a fraction as small as possible (i.e., $<5 \%$ ). Let the $r$-th realization of the path of $\{\phi\}_{t \geq 0}$ be denoted as $\left\{\phi_{r}\right\}_{t \geq 0}$ with $r=1, \ldots, N$. For each of these paths, the random variable that represents the 


\section{ETH}

Eidgenössische Technische Hochschule Zürich Swiss Federal Institute of Technology Zurich

http://www.sg.ethz.ch
P. Tasca, S. Battiston:

Market Procyclicality and Systemic Risk

Submitted March 16, 2013

exit time through the default boundary is defined as: $\tau_{r}=\inf \left\{t \geq 0: \phi_{r t} \geq 1\right\}$. The expected default time $\bar{\tau}(\phi)$ is approximated by $\hat{\tau}(\phi)$ as the total sum of the exit times divided by the number $N$ of paths:

$$
\hat{\tau}(\phi)=\frac{1}{N} \sum_{r}^{N} \tau_{r}\left(\mathbb{1}_{\phi_{r t}=1}\right) \approx \bar{\tau}(\phi)=\int_{0}^{T} t f_{\phi}\left(1, t \mid \phi_{0}, 0\right) d t .
$$

\section{References}

Acharya, V., 2009. A theory of systemic risk and design of prudential bank regulation. Journal of Financial Stability 5 (3), 224-255.

Adrian, T., Shin, H. S., 2008a. Financial intermediary leverage and value at risk. Federal Reserve Bank of New York Staff Reports 338.

Adrian, T., Shin, H. S., 2008b. Liquidity and financial cycles. BIS Working Papers 256.

Adrian, T., Shin, H. S., 2010. Liquidity and leverage. Journal of Financial Intermediation 19 (3), 418-437.

Adrian, T., Shin, H. S., 2011a. Financial intermediary balance sheet management. Federal Reserve Bank of New York, Staff Reports No. 532.

Adrian, T., Shin, H. S., 2011b. Procyclical leverage and value-at-risk. FRB of New York Staff Report No. 338.

Allen, F., Babus, A., Carletti, E., 2012. Asset commonality, debt maturity and systemic risk. Journal of Financial Economics 104 (3), 519-534.

Allen, F., Gale, D., Feb. 2001. Financial contagion. Journal of Political Economy 108 (1), 1-33.

Bargigli, L., Gallegati, M., Riccetti, L., Russo, A., 2012. Network analysis and calibration of the'leveraged network-based financial accelerator'. Available at SSRN 2160254.

Battiston, S., Gatti, D. D., Gallegati, M., Greenwald, B., Stiglitz, J. E., 2012a. Default cascades: When does risk diversification increase stability? Journal of Financial Stability 8 (3), 138 149.

Battiston, S., Gatti, D. D., Gallegati, M., Greenwald, B. C. N., Stiglitz, J. E., 2012b. Liaisons dangereuses: Increasing connectivity, risk sharing and systemic risk. Journal of Economic Dynamics and Control (forthcoming), early version NBER Working Paper Series n.15611. 


\section{ETH}

Eidgenössische Technische Hochschule Zürich Swiss Federal Institute of Technology Zurich

http://www.sg.ethz.ch
P. Tasca, S. Battiston:

Market Procyclicality and Systemic Risk

Submitted March 16, 2013

Battiston, S., Puliga, M., Kaushik, R., Tasca, P., Caldarelli, G., 2012c. Debtrank: Too central to fail? financial networks, the fed and systemic risk. Sci. Rep. 2 (541).

Bernanke, B., Lown, C., Friedman, B., 1991. The credit crunch. Brookings Papers on Economic Activity 1991 (2), 205-247.

BIS, Apr. 2010. International framework for liquidity risk measurement, standards and monitoring. Consultative document, Basel Committee on Banking Supervision, Bank for International Settlements, CH-4002 Basel, Switzerland.

Blum, J., Hellwig, M., 1995. The macroeconomic implications of capital adequacy requirements for banks. European Economic Review 39 (3-4), 739-749.

Brunnermeier, M., 2008. Deciphering the 2007-08 Liquidity and Credit Crunch. Journal of Economic Perspectives 23 (1), 77-100.

Calomiris, C., Wilson, B., 1998. Bank capital and portfolio management: The 1930's capital crunch and scramble to shed risk.

Cont, R., Moussa, A., Santos, E., 2011. Network structure and systemic risk in banking systems. New York.

EC, Jul. 2011. Proposal for a directive of the european parliament and of the cuncil. EU Directive $\operatorname{COM}(2011) 453$ final, DG Internal Market and Services, Banking and Financial Conglomerates Unit, European Commission, SPA2 4/29, B-1049 Brussels.

Elsinger, H., Lehar, A., Summer, M., 2006. Risk Assessment for Banking Systems. Management Science 52 (9), 1301-1314.

Estrella, A., 2004. The cyclical behavior of optimal bank capital. Journal of Banking \& Finance 28 (6), 1469-1498.

Freixas, X., Parigi, B. M., Rochet, J.-C., 2000. Systemic risk, interbank relations, and liquidity provision by the central bank. Journal of Money, Credit \& Banking 32, 611-638.

Furfine, C., 2003. Interbank Exposures: Quantifying the Risk of Contagion. Journal of Money, Credit \& Banking 35 (1), 111-129.

Gârleanu, N., Pedersen, L., 2007. Liquidity and risk management. The American Economic Review 97 (2), 193-197.

Greenlaw, D., Hatzius, J., Kashyap, A., Shin, H., 2008. Leveraged losses: lessons from the mortgage market meltdown. US Monetary Policy Forum Report No.2. 


\section{ETH}

Eidgenössische Technische Hochschule Zürich Swiss Federal Institute of Technology Zurich

http://www.sg.ethz.ch
P. Tasca, S. Battiston:

Market Procyclicality and Systemic Risk

Submitted March 16, 2013

Haldane, A., 2009. Rethinking the financial network. Speech delivered at the Financial Student Association, Amsterdam, April.

Iori, G., Jafarey, S., Padilla, F., 2006. Systemic risk on the interbank market. Journal of Economic Behaviour and Organization 61 (4), 525-542.

Kashyap, A., Stein, J., 2000. What do a million observations on banks say about the transmission of monetary policy? The American Economic Review, 407-428.

Kiyotaki, N., Moore, J., 2002. Balance-Sheet Contagion. American Economic Review 92 (2), $46-50$.

Krishnamurthy, A., 2010. Amplification mechanisms in liquidity crises. American Economic Journal: Macroeconomics 2 (3), 1-30.

Shin, H., 2008. Risk and liquidity in a system context. Journal of Financial Intermediation 17 (3), 315-329.

Stiglitz, J., Weiss, A., 1981. Credit rationing in markets with imperfect information. The American economic review 71 (3), 393-410.

Tasca, P., Battiston, S., 2011. Diversification and financial stability. Working Paper Series CCSS11-001.

Vitali, S., Glattfelder, J., Battiston, S., 2011. The network of global corporate control. PloS one 6 (10), e25995. 